\title{
Study of aerodynamic forces acting on a train using a tornado simulator
}

\author{
Masahiro SUZUKI* and Nobuyuki OKURA* \\ *Department of Vehicle and Mechanical Engineering, Meijo University \\ 1-501 Shiogamaguchi, Tempaku-ku, Nagoya-shi, Aichi 468-8502, Japan \\ E-mail: msuzuki@meijo-u.ac.jp
}

Received 2 September 2016

\begin{abstract}
A novel experiment was conducted to investigate the aerodynamic forces acting on a train traveling through a tornado, in which we developed a moving model rig with a tornado simulator. The flow field generated by the tornado simulator was validated by comparison with those of real tornadoes and the Rankine vortex model. Using this setup, we measured unsteady surface pressures on a model train as it passed through the vortex center. The side force, lift force, and yawing moment were estimated from the pressure data. The results were as follows: 1) the side force acting on the train changed its direction from negative to positive while passing through the tornado-like swirling flow; 2) the lift force increased as the train approached the flow and became maximum around the vortex center; 3 ) the yawing moment first decreased slightly and then reached its maximum around the vortex center. Asymmetric wave forms of the forces and moment at the center of the tornado simulator suggested that the train itself may have affected the vortex structure of the flow.
\end{abstract}

Key words : Railway, Train, Tornado, Aerodynamic force, Tornado simulator, Moving model rig, Rankin vortex model

\section{Introduction}

A tornado is a weather phenomenon with a destructively rotating column of air that stretches from a convective cloud such as a cumulus or cumulonimbus to the ground (Rotunno, 2013). They can cause serious damage, sometimes with casualties. Houses and buildings are often destroyed, and cars and trucks are frequently overturned. There is also a possibility for trains to overturn. In fact, at least three train-turnover accidents in Japan are suspected to have been caused by tornadoes; they are as follows. In 1978, a train of the Touzai line flipped over on the Arakawa-Nakagawa Bridge in Tokyo. A band of destroyed houses stretching across the surrounding area were suggested to have been destroyed by a tornado (Japan Meteorological Agency, online). In addition, an express train of the JR Uetsu line was overturned by a gust of wind in Yamagata prefecture in 2006. An accident analysis report suggested that a tornado or a downburst probably generated the gust (Aircraft and Railway Accidents Investigation Commission, 2008a). Furthermore, a train of the JR Nippo line was overturned by a tornado in Miyazaki prefecture in 2006. The tornado was rated at F2 (wind speed: $50-69 \mathrm{~m} / \mathrm{s}$ ) on the Fujita scale based on the damage to the surrounding area (Aircraft and Railway Accidents Investigation Commission, 2008b).

Although studies on tornado phenomenology and climatology have been vigorously conducted, a complete picture of the tornado phenomena is yet to be obtained. Their unpredictable and dangerous nature makes field studies difficult, therefore experimental and numerical approaches are a practical alternative. Several laboratory experiments have been modeled after the pioneering study of Ward (1972), in which vortices were generated with features similar to those observed in nature. Recently, Hann et al. (2008) presented a tornado simulator for the purpose of estimating the aerodynamic effects of tornadoes on civil engineering structures. They validated the flow structures by comparing them 
with the mobile Doppler radar observations of two major tornadoes.

Crosswind effects on trains have been studied extensively for decades. A large number of wind tunnel experiments have been conducted to investigate the aerodynamic forces acting on trains. Although a train is normally placed in a wind tunnel (CEN, 2010), several studies have run a train in front of a wind tunnel by using a moving model rig in order to investigate the effect of the relative velocity between the train and the track (Baker, 1986).

However, there are almost no studies dealing with the aerodynamic forces acting on a train by a tornado, although train turnover accidents caused by tornadoes have occurred in the past. Therefore, we have developed an experimental method to investigate the aerodynamic forces acting on the train in a tornado by using a moving model rig with a tornado simulator.

\section{Method}

Our experimental system consisted of a moving model rig and a tornado simulator. The tornado simulator generated a tornado-like swirling flow, and the moving model rig allowed a model train to run through the swirling flow. Figure 1 illustrates the main components of the setup.

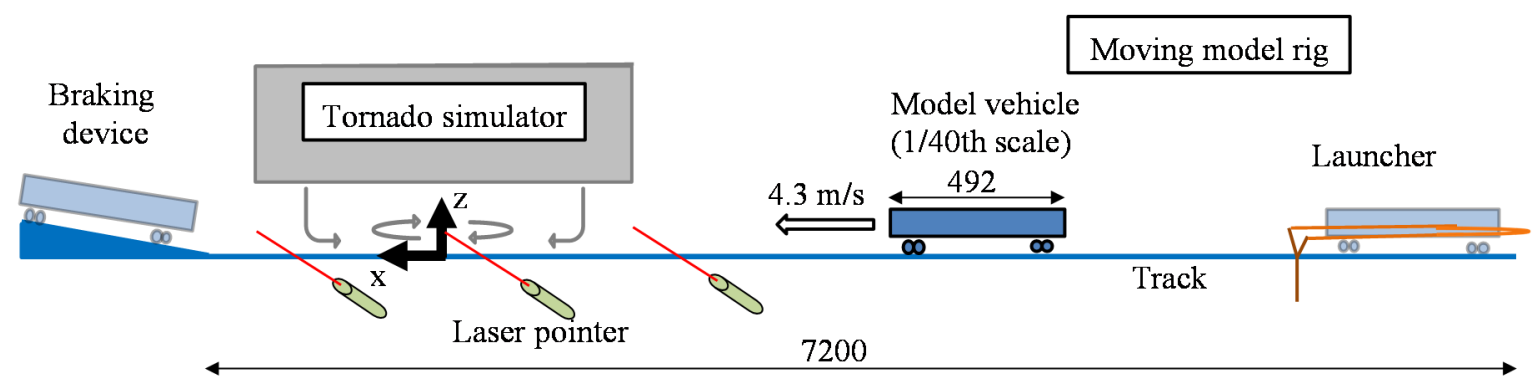

Fig. 1 Experimental setup (units: $\mathrm{mm}$ )

\subsection{Tornado simulator}

Our tornado simulator was designed on the basis of the one at Iowa State University (Haan et al., 2008). This simulator consisted of inner and outer ducts, a fan, guide vanes, and a stage (Fig. 2). The fan made an updraft in the inner duct. The flow then went through the guide vanes at the top of the simulator. Since the guide vanes were attached at an angle, they created a twisted downflow in the outer duct. This process generated a tornado-like swirling flow on the stage.

The diameter of the fan was $750 \mathrm{~mm}$ and its rotational speed was 1,660 rpm. The outside and inside diameters of the outer duct were $1,720 \mathrm{~mm}$ and $1,520 \mathrm{~mm}$, respectively. The diameter of the inner duct was $800 \mathrm{~mm}$, and the height of both ducts was $600 \mathrm{~mm}$. The distance from the stage to the ducts (height of the convergence layer, $H$ in Fig. 2) was $300 \mathrm{~mm}$. 18 guide vanes were installed around the top of the simulator at regular intervals. Their angle could be changed between $30^{\circ}$ and $70^{\circ}$; we set it at $50^{\circ}$ for the experiment.

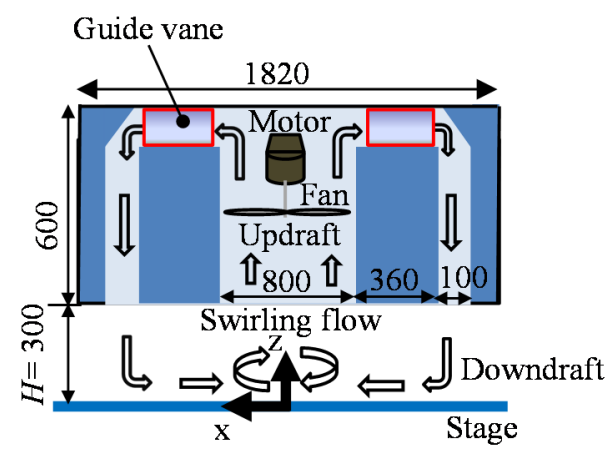

(a) Cross-sectional view

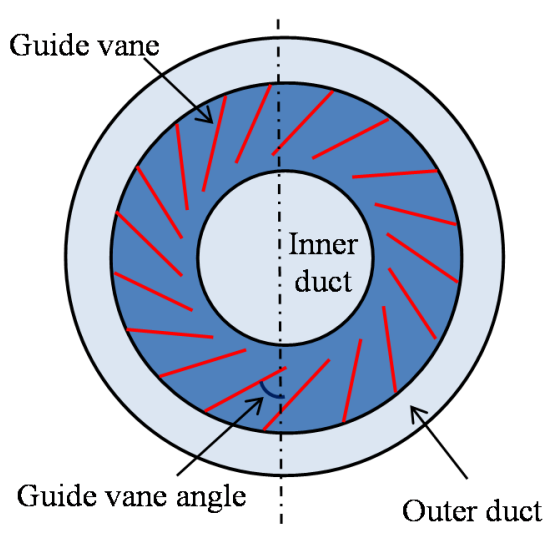

(b) Top view of inner and outer ducts and guide vanes

Fig. 2 Tornado simulator (units: $\mathrm{mm}$ ) 
The swirl ratio is an important parameter for determining the structure of a tornado (Church et al., 1979). It is expressed as the ratio of the angular momentum to the updraft momentum as follows:

$$
\mathrm{S}=\frac{r}{2 H} \tan \theta
$$

where $r$ is the radius of the inner duct, $H$ is the height of the convergence layer, and $\theta$ is the guide vane angle. The swirl ratio in our experiment was 0.8 , while the swirl ratios of natural tornadoes are estimated at 0.05-2 (Church et al., 1979).

\subsection{Moving model rig}

The moving model rig consisted of a straight track, a launcher, a breaking device, and a 1/40 scale, self-propelled model vehicle (Fig. 1). The length of the track was 7,200 mm. It was placed in such a way that the train passed though the center of the swirling flow. The launcher gave the train an initial velocity of $4.3 \mathrm{~m} / \mathrm{s}$ by using an elastic force provided by rubber bands. After the launch, the train traveled on the track while the speed was maintained by a motor installed on the vehicle. The braking device stopped the train safely.

The model train contained pressure sensors to measure unsteady surface pressures during its run through the swirling flow. There were 72 pressure ports on the vehicle surfaces (Fig. 3). A 12-channel data logger with an on-board AD converter recorded unsteady pressure data. The sampling rate was $10 \mathrm{kHz}$. Unsteady aerodynamic forces acting on the vehicle were estimated from the pressure data. A phototransistor and laser pointers were used to detect the time when the train crosses the tornado simulator. The phototransistor installed on the train surface reacted to laser beams emitted from the laser pointers. Three laser beams were set at the entrance, center, and exit of the tornado simulator. Figure 4 defines the directions of the aerodynamic forces and moment acting on the train.
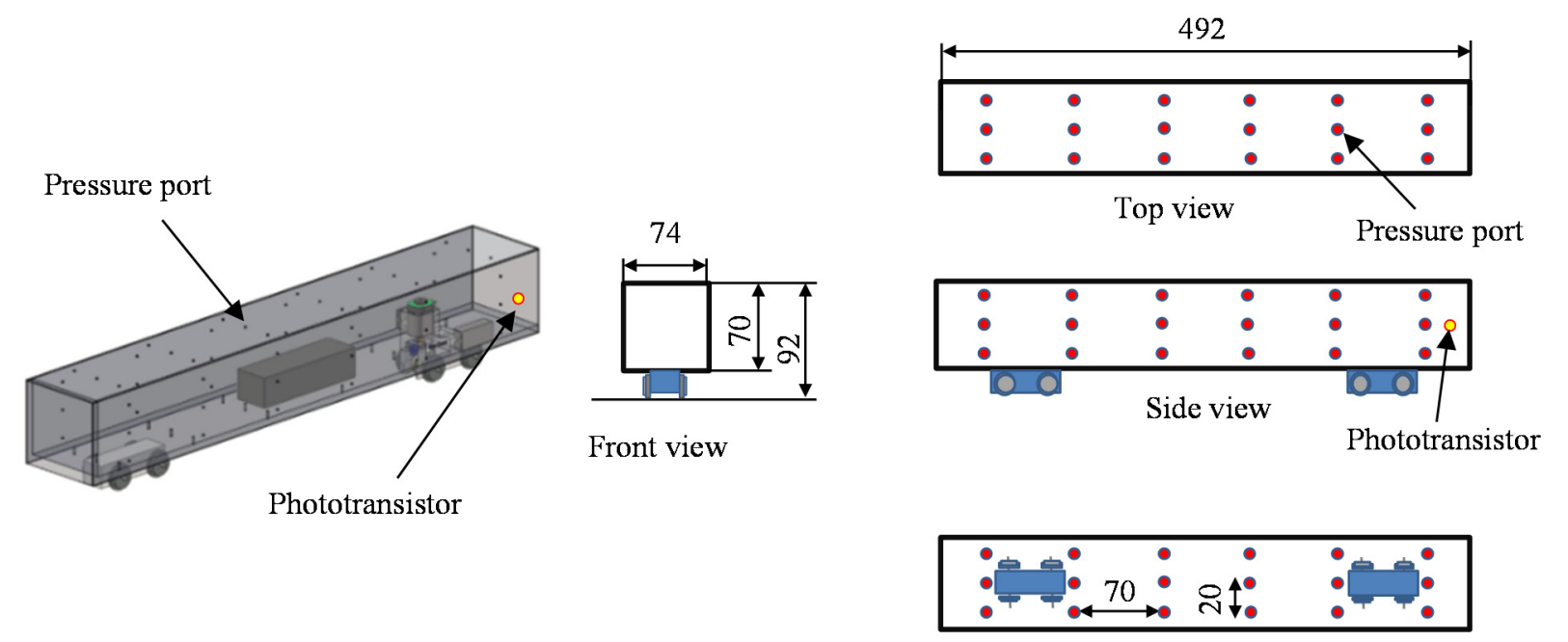

Bottom view

Fig. 3 Pressure ports and a phototransistor on the model train (units: $\mathrm{mm}$ )

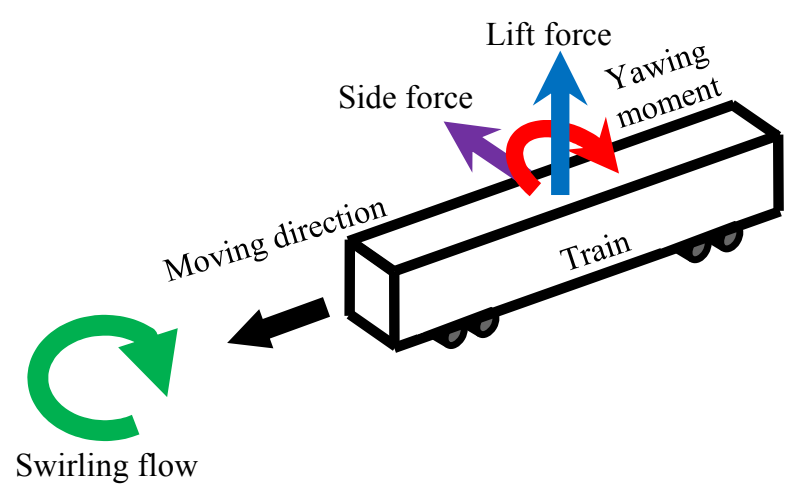

Fig. 4 Definition of side and lift forces and yawing moment acting on the train and rotational direction of the swirling flow 


\section{Results and discussion}

\subsection{Performance of the tornado simulator}

First, the pressure and tangential velocity distributions on the stage were measured to evaluate the performance of the tornado simulator. Pressure ports were set radially on the stage at $25 \mathrm{~mm}$ intervals around the center. More pressure ports were set at $50 \mathrm{~mm}$ intervals outside the center area. As a first and simple step for measuring the velocity distribution, a Pitot tube was inserted at the center height of the train in the tangential direction to the swirling flow.

Figure 5 shows the tangential velocity and pressure distribution. The horizontal axis indicates the distance from the center of the tornado simulator. A blue line displays the theoretical value of the Rankine vortex model—a simple model of a tornado that is widely used (American Nuclear Society, 2011). This model has a tangential velocity distribution that is proportional to the distance from the center in the inner region of the vortex. In the outer region, it is inversely proportional to the distance. In the Rankine model, tangential velocity $V$ and pressure $p$ are expressed as follows:

$$
\begin{aligned}
& V(x)=\left\{\begin{array}{l}
V_{\max } \frac{|x|}{R},|x| \leq R \\
V_{\max } \frac{R}{|x|},|x|>R
\end{array}\right. \\
& p(x)=\left\{\begin{array}{c}
\frac{1}{2} p_{\min }\left[2-\frac{x^{2}}{R^{2}}\right],|x| \leq R \\
\frac{1}{2} p_{\min } \frac{R^{2}}{x^{2}},|x|>R
\end{array}\right.
\end{aligned}
$$

where, $x$ is the distance from the tornado center, $V_{\text {max }}$ is the maximum tangential velocity, $R$ is the core radius, and $p_{\min }$ is the minimum pressure. There was good agreement between our measured values and that of the Rankine vortex model. The maximum tangential velocity is $\sim 8 \mathrm{~m} / \mathrm{s}$, which is almost twice the train speed. If the flow is insensitive to Reynolds number, this ratio is maintained in the real world. For example, suppose the train speed is $60 \mathrm{~km} / \mathrm{h}(16.7 \mathrm{~m} / \mathrm{s})$, then the maximum tangential velocity becomes $33.4 \mathrm{~m} / \mathrm{s}$. This situation can happen in the real world. However, the Reynolds number dependence of the flow should be investigated in the future work. The core radius is about $100 \mathrm{~mm}$, which is 20 percent of the train length and corresponds to $4 \mathrm{~m}$ in full scale. According to statistics of tornadoes from Jan. 1961 to Oct. 2008 in Japan, the width of the damage area by the tornado are mainly distributed over tens of meters and there are some below ten meters (Tokyo Polytechnic University, 2011). Considering the core radius is smaller than the width of the damage area, a scenario that a train meets a small tornado like our experiment is not unrealistic. Based on the maximum tangential velocity and the train width, the Reynolds number is $3.8 \times 10^{4}$. The Pitot tube measurement may have some errors because the flow is a turbulent swirling flow. More accurate measurements like PIV should be conducted in the future.

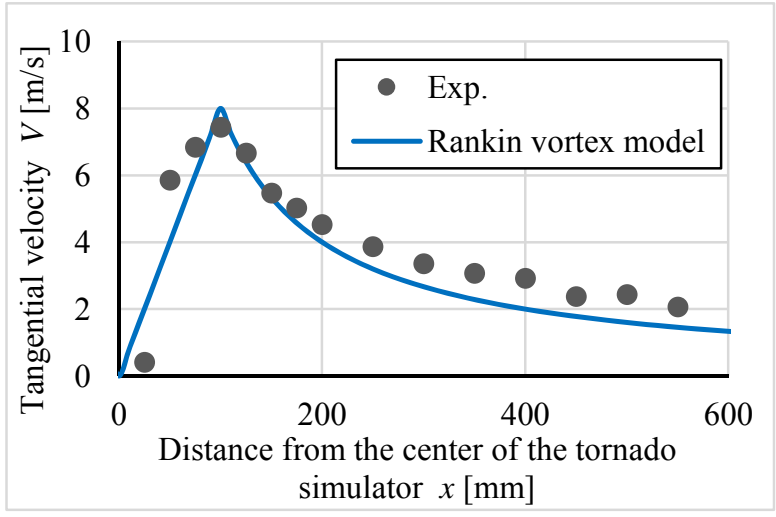

(a) Tangential velocity distribution

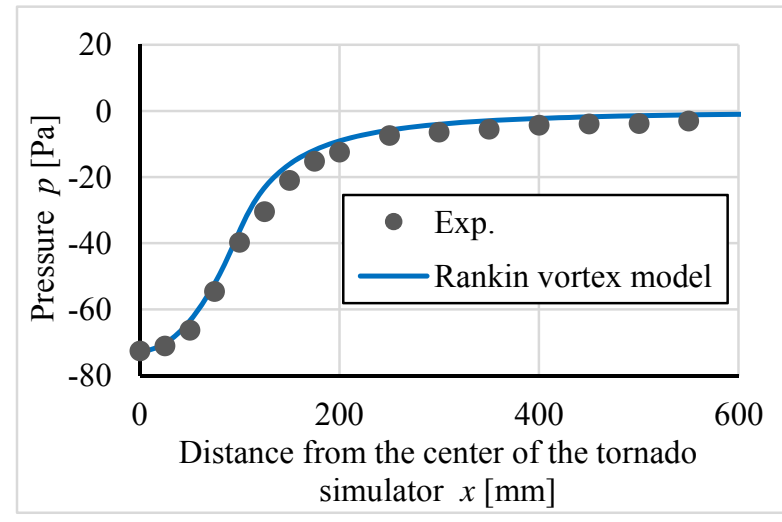

(b) Pressure distribution

Fig. 5 Performance of the tornado simulator 
Figure 6 shows normalized tangential velocity profiles to compare with those of the real tornadoes. The distance from the vortex centre, $x$, is normalized by the core radius $R$, and the velocity by the maximum tangential velocity $V_{\text {max }}$. Doppler radar data from full-scale tornados at Spencer, South Dakota and Mulhall, Oklahoma which were compiled by Haan et al. (2008) are plotted with the present result in the figure. The height of the horizontal plane of these full-scale tornado data is $Z=0.52 R$ where the ground plane is $Z=0$, while ours is $Z=0.54 R$. Our result agrees well with the radar data.

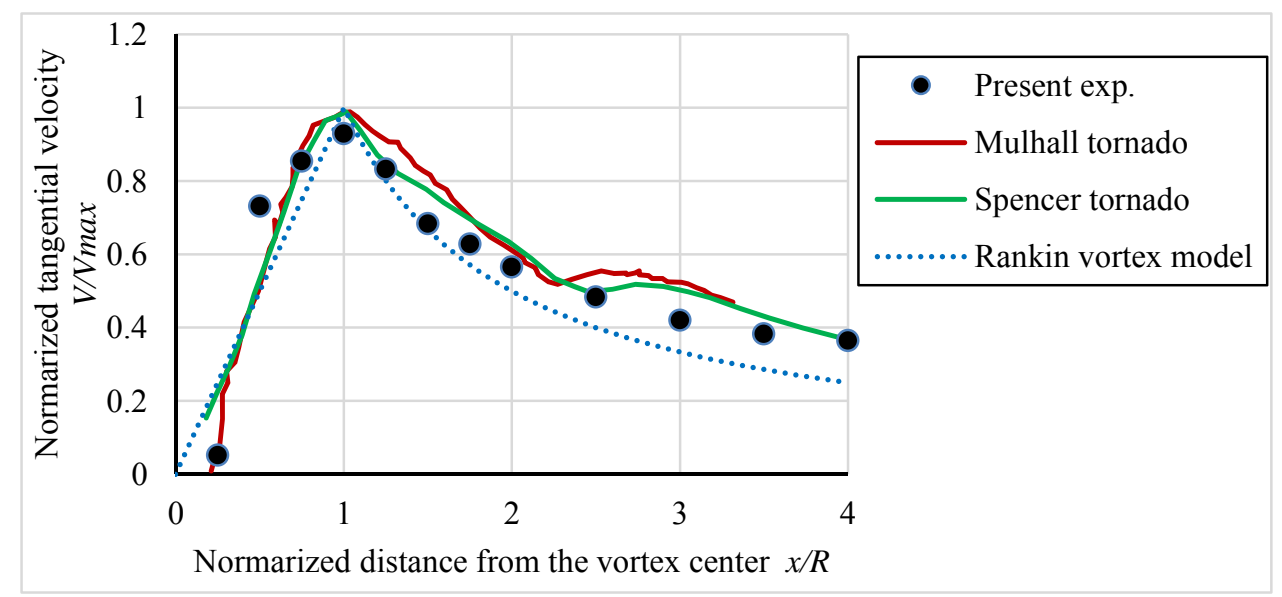

Fig. 6 Comparison of velocity distributions with those of the real tornadoes

\subsection{Aerodynamic forces acting on the train}

The train was run 30 times to obtain the ensemble averaged pressure data for each pressure port. The side force $F_{S}$, the lift force $F_{l}$, and the yawing moment $M_{y}$ were estimated from the pressure data. The pressure coefficient $\mathrm{C}_{\mathrm{p}}$, the side force coefficient $C_{s}$, the lift force coefficient $C_{l}$, and the yawing moment coefficient $C_{y}$ are defined as follows:

$$
\begin{aligned}
& \mathrm{C}_{\mathrm{p}}(t)=\frac{p(t)-p_{0}}{\frac{1}{2} \rho V_{\text {max }}{ }^{2}}, \\
& \mathrm{C}_{\mathrm{s}}(t)=\frac{F_{S}(t)}{\frac{1}{2} \rho V_{\text {max }^{2} S_{a}}}, \\
& \mathrm{C}_{\mathrm{l}}(t)=\frac{F_{l}(t)}{\frac{1}{2} \rho V_{\max ^{2} S_{b}}}, \\
& \mathrm{C}_{\mathrm{y}}(t)=\frac{M_{y}(t)}{\frac{1}{2} \rho V_{\text {max }^{2} S_{a} l}} .
\end{aligned}
$$

Here, $p_{0}$ is the atmospheric pressure, $\rho$ is the density of air, $V_{\max }$ is the maximum tangential velocity $(8 \mathrm{~m} / \mathrm{s}), t$ is time, $S_{a}$ is the area of the lateral side of the train $\left(0.034 \mathrm{~m}^{2}\right), S_{b}$ is the area of the top surface of the train $\left(0.036 \mathrm{~m}^{2}\right)$, and $l$ is half the train length $(0.246 \mathrm{~m})$.

Figure 7 shows the ensemble averaged time histories of pressure coefficients at the right, left, top, and bottom sides of the train as the vehicle runs though the swirling flow. The horizontal axis indicates the position of the train center, normalized by the core radius $R$. Note that the center of the tornado simulator is located at $x / R=0$. On the right, left, and top sides of the train, the pressure fluctuation travels downstream from Sections 1 to 3 . The pressure fluctuation is the greatest at Section 1. On the bottom side of the train, the pressure fluctuation patterns differ between the sections. Although the pressures at pressure port no. B1 and B3 fluctuate independently of one another, the pressure at no. B2 stays at nearly atmospheric pressure. Bogies may cause these complex pressure patterns.

The bold black lines in Fig. 8 display the ensemble averaged time histories of the side force, lift force, and yawing 
moment coefficients. First, a negative side force acts on the train. It then becomes positive while passing through the tornado-like swirling flow. The side force switches from negative to positive before the train center reaches the center of the tornado simulator. The absolute values of the negative and positive peaks are almost identical. A negative peak occurs at $x / R=-2.8$, and a positive one occurs at $x / R=2.3$.

The lift force increases as the train approaches the vortex center and reaches its maximum at the center of the tornado simulator. The waveform is not symmetrical at the center of the tornado simulator. More lift force acts on the train at $x / R$ $<0$. The lift force decreases locally at $x / R=-1.2$ and 2.7 .

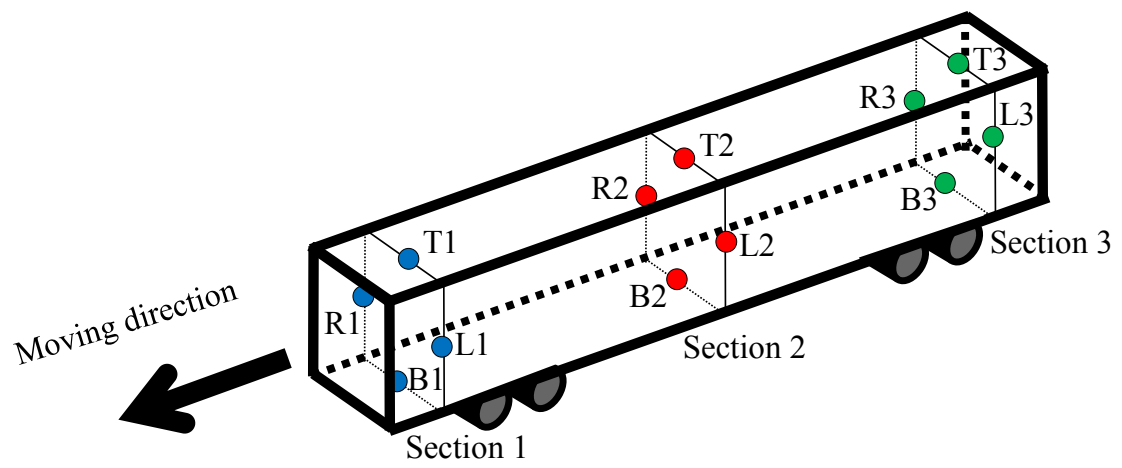

(a) Positions of pressure ports

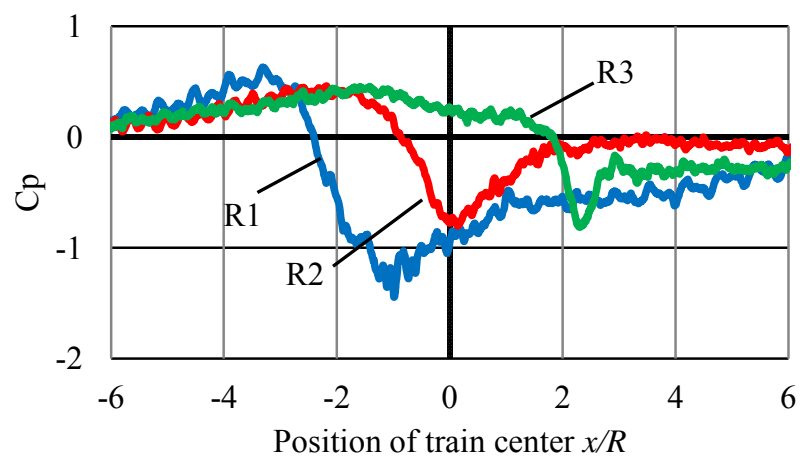

(b) Right side of the train

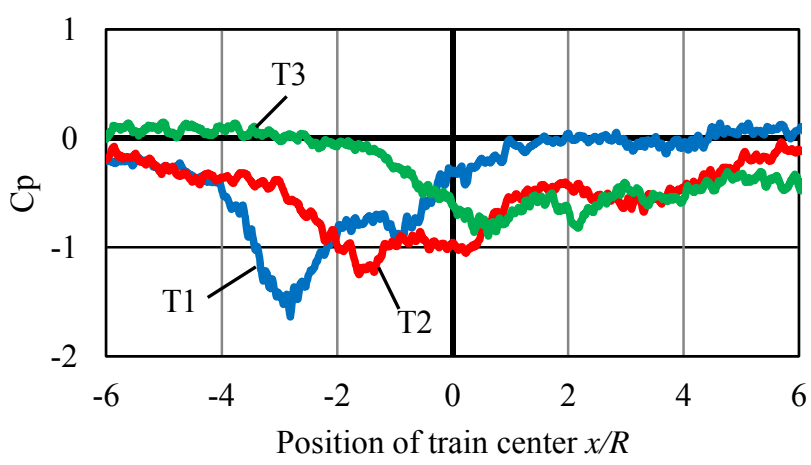

(d) Top side of the train

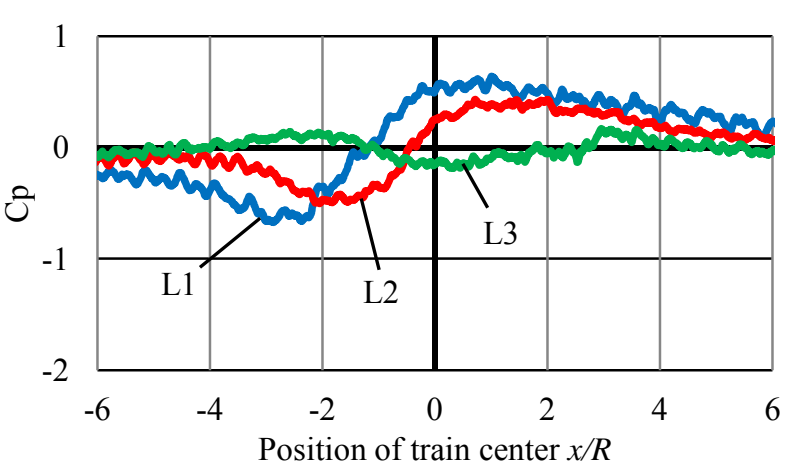

(c) Left side of the train

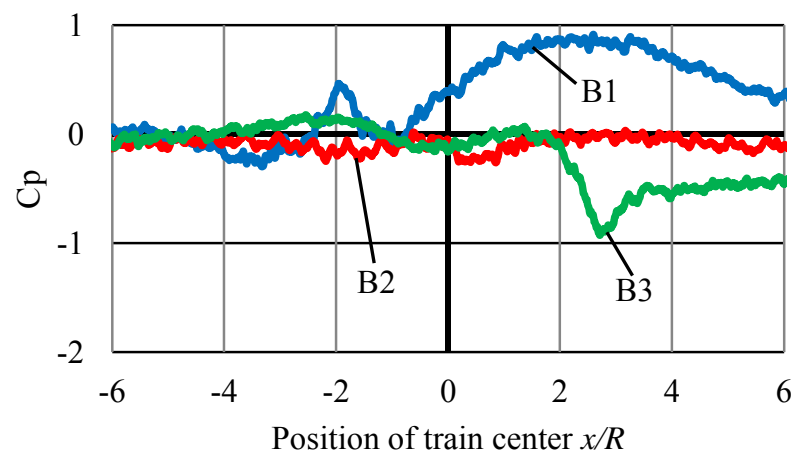

(e) Bottom side of the train

Fig. 7 Ensemble averaged time histories of pressures on the right, left, top and bottom sides of the train

A negative yawing moment also acts on the train first. The negative peak is at $x / R=-2.8$; this position coincides with that of the side force peak. The yawing moment then increases and reaches its maximum just before the train center reaches the center of the tornado simulator. It decreases to the minimum at $x / R=2.3$, which coincides with the position of the positive peak of the side force, and it then increases slightly and diminishes gradually.

The thin blue lines in Fig. 8 represent the values estimated on the assumption that the aerodynamic forces acting on 
each section of the train are proportional to the square of the tangential velocity obtained using the Rankine vortex model:

$$
\begin{gathered}
\Delta F_{s}=\mathrm{k}_{s} V_{R}^{2} \Delta S_{a}, \\
\Delta F_{l}=\mathrm{k}_{l} V_{R}^{2} \Delta S_{b} .
\end{gathered}
$$

Here, $\Delta F_{S}$ and $\Delta F_{l}$ are side and lift forces acting on each section of the train, $V_{R}$ is the tangential velocity at the location of the train section obtained by the Rankine vortex model, and $\mathrm{k}_{s}$ and $\mathrm{k}_{l}$ are the proportionality constants. The side and lift forces acting on the whole train are obtained by integrating Eqs. (8) and (9). The proportionality constants $\mathrm{k}_{s}$ and $\mathrm{k}_{l}$ are set in such a way that the minimum value of the side force and the maximum value of lift force agree with those of the experiment, respectively. The yawing moment acting on the whole train is calculated by multiplying Eq. (8) by the distance between the train center and the section and integrating the resultant equation.

The peak positions of the forces and yawing moment, estimated based on the Rankine vortex model, are in fairly close agreement with the experimental results. In spite of setting the proportionality constant $\mathrm{k}_{S}$ in such a way that the peak values of the side force agree with those of the experiment, the estimated yawing moment is smaller than the experimental result. This suggests that greater forces act near the edge of the train. The discrepancy between the estimated and experimental values of the lift force waveforms also suggests that the effect of the updraft, which is excluded from the Rankine vortex model, is dominant on the lift force. The asymmetric wave forms of the forces and moment at the center of the tornado simulator suggest that the vehicle itself may affect the vortex structure of the tornado-like swirling flow.

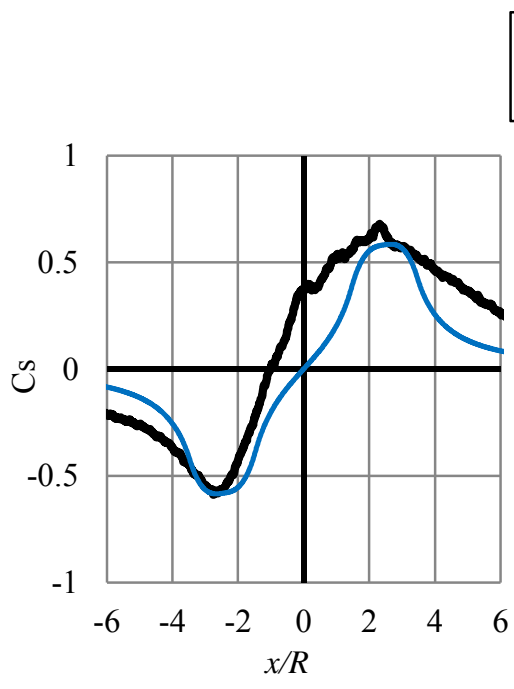

(a) Side force

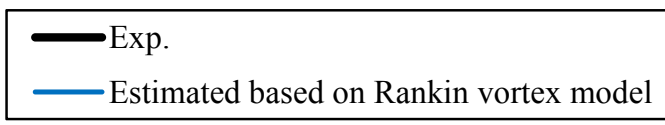

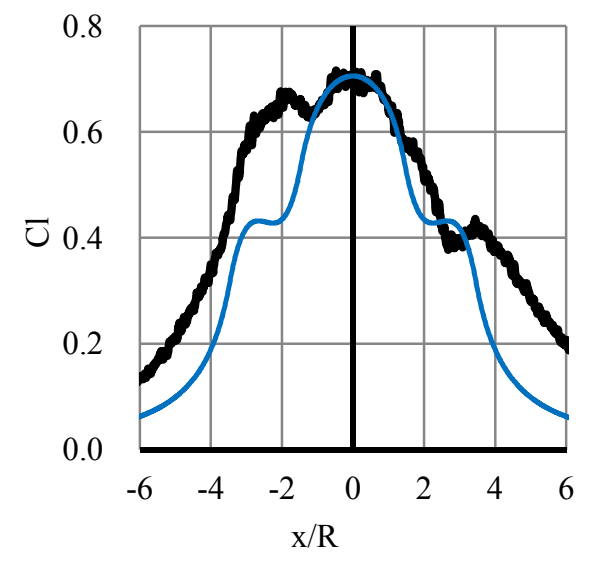

(b) Lift force

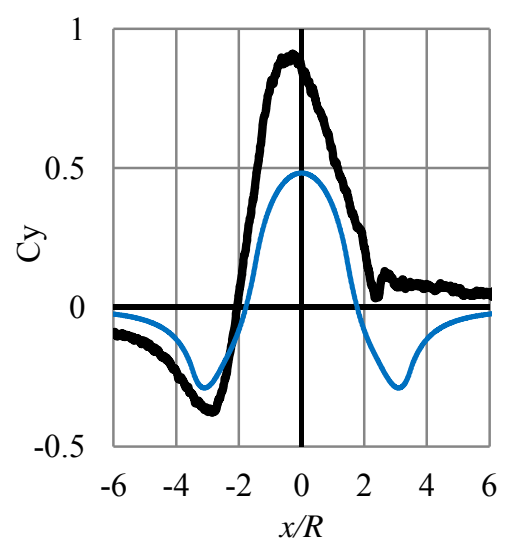

(c) Yawing moment

Fig. 8 Ensemble averaged time histories of side and lift forces, and yawing moment

\section{Conclusion}

We developed an experimental method to investigate the aerodynamic forces acting on a train passing through a tornado. This was performed using a moving model rig and a tornado simulator. The flow field generated by the tornado simulator was validated using the full-scale data and the Rankine vortex model. The moving model rig enabled the measurement of the unsteady aerodynamic forces that occurred when the train ran under the tornado-like swirl flow field. The results show that the aerodynamic forces changed relative to the position of the train. Their asymmetric waveforms at the center of the tornado simulator suggest that the train itself may have affected the vortex structure of the tornadolike swirling flow.

Because this was the first trial using the facility, several questions still remain unanswered. These questions are as follows: what is the detailed flow field around a train? what are the effects of a tornado's size, structure, and translation 
speed? what are the effects of a train's size, speed, and number of cars in the train set? what are the effects of ground configurations? Further studies are required to answer these questions.

\section{Acknowledgement}

The authors thank Yuki Nakamura and Toshiaki Moroto for their help conducting the experiments. This work was supported by JSPS KAKEN Grant Number 26350491.

\section{References}

Aircraft and Railway Accidents Investigation Commission, Railway accident analysis report RA2008-4 (2008a) (in Japanese)

Aircraft and Railway Accidents Investigation Commission, Railway accident analysis report RA2008-6 (2008b) (in Japanese)

American Nuclear Society, Estimating tornado, hurricane, and extreme straight line wind characteristics at nuclear facility sites: An American national standard ANSI/ANS-2.3-2011 (2011).

Baker, C. J., Train aerodynamic forces and moments from moving model experiments, Journal of Wind Engineering and Industrial Aerodynamics, Vol. 24, No. 3 (1986), pp.227-251.

CEN, Railway applications-Aerodynamics-Part 6: Requirements and test procedures for cross wind assessment, EN14067-6 (2010).

Church, C. R., Snow, J. T., Baker, G. L., Agee, E. M., 1979. Characteristics of tornado-like vortices as a function of swirl ratio: A laboratory investigation, Journal of the Atmospheric Sciences, Vol. 36 (1979), pp. 1755-1776.

Haan, F. L., Sarkar, P. P., Gallus, W. A., Design, construction, and performance of a large tornado simulator for wind engineering applications, Engineering Structures, Vol. 30 (2008), pp. 1146-1159.

Japan Meteorological Agency, Tornado data base (online), available from $<$ http://www.data.jma.go.jp/obd/stats/data/bosai/tornado/1978022801/list.html>, (accessed on 4 August, 2016) (in Japanese)

Rotunno, R., The fluid dynamics of tornadoes, Annual Review of Fluid Mechanics, Vol. 45 (2013), pp. 59-84.

Tokyo Polytechnic University, Study on tornado effect on nuclear power plants, Contracted survey research report to Japan Nuclear Energy Safety Organization (2011) (in Japanese)

Ward, N. B., The exploration of certain features of tornado dynamics using a laboratory model, Journal of the Atmospheric Sciences, Vol. 29 (1972), pp. 1194-1204. 